\title{
Profinite groups in which centralizers are virtually procyclic
}

\author{
Pavel Shumyatsky and Pavel Zalesskii
}

\begin{abstract}
The article deals with profinite groups in which centralizers are virtually procyclic. Suppose that $G$ is a profinite group such that the centralizer of every nontrivial element is virtually torsion-free while the centralizer of every element of infinite order is virtually procyclic. We show that $G$ is either virtually pro- $p$ for some prime $p$ or virtually torsion-free procyclic (Theorem 1.1). The same conclusion holds for profinite groups in which the centralizer of every nontrivial element is virtually procyclic (Theorem 1.2); moreover, if $G$ is not pro- $p$, then $G$ has finite rank.
\end{abstract}

\section{Introduction}

Centralizers play a fundamental role in group theory. In particular, centralizers were of crucial importance in the development of the theories of finite and locally finite groups (cf. [4], [7], and [6]). On the other hand, results on centralizers in profinite groups are still fairly scarce.

In this paper our theme is as follows. Given an information on centralizers of nontrivial elements of a profinite group $G$, what can be deduced about the structure of $G$ ?

This study started in [13] where the authors handled profinite groups with abelian centralizers. Later the work was extended by the first author to profinite groups in which the centralizers are pronilpotent [14].

The next condition on centralizers comes from hyperbolic groups that are known to have very restricted centralizes. Namely, a residually finite hyperbolic group is virtually torsion free (see [10, Theorem 5.1])

1991 Mathematics Subject Classification. 20E18.

Key words and phrases. Profinite groups, procyclic groups, centralizers.

This research was supported by CNPq and FAPDF. 
and the centralizer of an element of infinite order of it is virtually cyclic (see [1, Proposition 3.5]). Imposing this property on the centralizers of elements of profinite groups we show that a 'hyperbolic-like' profinite group must be either virtually pro- $p$ or virtually procyclic. Note that in general a procyclic group is not necessarily virtually torsion free (cf. Remark 3.2).

THEOREM 1.1. Let $G$ be a profinite group such that the centralizer of every nontrivial element is virtually torsion-free while the centralizer of every element of infinite order is virtually procyclic. Then $G$ is either virtually pro-p for some prime $p$ or virtually torsion-free procyclic.

Having dealt with Theorem 1.1, it was natural to look at profinite groups in which the centralizer of every nontrivial element is virtually procyclic.

THEOREM 1.2. Let $G$ be a profinite group in which the centralizer of every nontrivial element is virtually procyclic. Then $G$ is either virtually pro-p for some prime $p$ or virtually procyclic.

Recall that a profinite group $K$ is said to have finite (Prüfer) rank $r$ if every subgroup of $K$ can be generated by $r$ elements. The next corollary can be easily deduced from Theorem 1.2 .

COROLlaRY 1.3. Let $G$ be a profinite group in which the centralizer of every nontrivial element is virtually procyclic and suppose that $G$ is not a pro-p group. Then $G$ has finite rank.

In view of the above corollary the following conjecture seems plausible.

CONJECTURE 1.4. Let $G$ be a profinite group in which the centralizer of every nontrivial element has finite rank. Suppose that $G$ is not a pro-p group. Then $G$ has finite rank.

Note that the results obtained in [14] show that the conjecture is

correct if we additionally assume that the centralizers in $G$ are pronilpotent.

The proofs of both Theorem 1.1 and Theorem 1.2 depend on the classification of finite simple groups.

\section{Preliminaries}

We start this section by quoting the following famous theorem of Zelmanov [17].

THEOREM 2.1. Each compact torsion group is locally finite. 
Recall that according to the Hall-Kulatilaka theorem [5] each infinite locally finite group has an infinite abelian subgroup. Combining this with Theorem 2.1 we deduce

THEOREM 2.2. Each infinite locally finite, or profinite, group has an infinite abelian subgroup.

If $A$ is a group of automorphisms of a group $G$, the subgroup generated by all elements of the form $g^{-1} g^{\alpha}$ with $g \in G$ and $\alpha \in A$ is denoted by $[G, A]$. It is well-known that the subgroup $[G, A]$ is an $A$-invariant normal subgroup in $G$. We write $C_{G}(A)$ for the centralizer of $A$ in $G$.

The next lemma is a list of useful facts on coprime actions. Here $|K|$ means the order of a profinite group $K$ (see for example [11] for the definition of the order of a profinite group). For finite groups the lemma is well known (see for example [4, Ch. 5 and 6]). For infinite profinite groups the lemma follows from the case of finite groups and the inverse limit argument (see [11, Proposition 2.3.16] for a detailed proof of item (iii)). As usual, $\pi(G)$ denotes the set of prime divisors of the order of $G$. By an automorphism of a profinite group we always mean a continuous automorphism.

LEMMA 2.3. Let $A$ be a profinite group of automorphisms of a profinite group $G$ such that $(|G|,|A|)=1$. Then

(i) $G=[G, A] C_{G}(A)$.

(ii) $[G, A, A]=[G, A]$.

(iii) $C_{G / N}(A)=N C_{G}(A) / N$ for any $A$-invariant normal subgroup $N$ of $G$.

(iv) $G$ contains an A-invariant Sylow q-subgroup for each prime $q \in \pi(G)$.

Throughout the paper we denote by $\langle X\rangle$ the (sub)group generated by the set $X$.

LEMMA 2.4. Let $A$ be an infinite procyclic group acting coprimely on a profinite group $G$. Let $A_{i}=A^{i !}$ denote the subgroup generated by the $i$ !th powers of elements of $A$. Set $G_{i}=C_{G}\left(A_{i}\right)$. For each $A$ invariant open normal subgroup $N$ of $G$ there is $i$ such that $G=N G_{i}$. If there is an index $j$ such that $G_{j}=G_{j+k}$ for each $k=1,2 \ldots$, then $G_{j}=G$.

Proof. Let $N$ be an $A$-invariant open normal subgroup of $G$. Obviously, $A$ induces a finite group of automorphisms of $G / N$. Thus, there exists $i$ such that $A_{i}$ acts on $G / N$ trivially. In view of Lemma 2.3 (iii) it follows that $G=N G_{i}$. 
Now suppose that there is an index $j$ such that $G_{j}=G_{j+k}$ for each $k=1,2 \ldots$ Suppose that $G_{j} \neq G$. Then there exists an $A$-invariant open normal subgroup $N$ of $G$ such that $N G_{j} \neq G$. On the other hand, by the above, there is an index $i$ such that $G=N G_{i}$. Note that we have containments

$$
G_{1} \leq G_{2} \leq \cdots \leq G_{u} \leq G_{u+1} \leq \cdots
$$

Since by assumptions $G_{j}=G_{j+k}$ for each $k=1,2 \ldots$, it follows that $G_{i} \leq G_{j}$ and so $N G_{j}=G$, a contradiction.

The next lemma uses the well-known corollary of the classification of finite simple groups that the order of any nonabelian finite simple group is divisible by 3 or 5 .

LEMMA 2.5. Let $H$ be a profinite group in which for all odd primes $p \in \pi(G)$ the Sylow p-subgroups are finite. Then $H$ is virtually prosoluble.

Proof. Suppose that the lemma is false. Choose an open $\{3,5\}^{\prime}-$ subgroup $K \leq G$. We see that $K$ is prosoluble, a contradiction.

Throughout the paper we write $O_{\pi}(G)$ for the maximal normal pro- $\pi$ subgroup of the group $G$. As usual, the additive group of $p$-adic integers is denoted by $\mathbb{Z}_{p}$. Recall that the group of automorphisms of $\mathbb{Z}_{p}$ is isomorphic to $\mathbb{Z}_{p} \oplus C_{p-1}$ if $p \geq 3$ and $\mathbb{Z}_{2} \oplus C_{2}$ if $p=2$ (see for example [11, Theorem 4.4.7]). Here $C_{n}$ stands for the cyclic group of order $n$. Consequently, the group of automorphisms of any procyclic group is abelian.

LEMMA 2.6. Let $G$ be a virtually procyclic profinite group. The second derived group $G^{\prime \prime}$ is finite.

Proof. Let $K$ be an open normal procyclic subgroup of $G$. Observe that $G^{\prime} \leq C_{G}(K)$. It follows that the centre of $G^{\prime}$ has finite index, whence by Schur's theorem [12, Theorem 10.1.4] $G^{\prime \prime}$ is finite.

Lemma 2.7. Let $G$ be a profinite group whose Sylow 2-subgroups are virtually procyclic. Then $G$ is virtually prosoluble.

Proof. If the Sylow 2-subgroups of $G$ are finite, then $G$ has an open normal 2 -subgroup which is prosoluble by the Feit-Thompson theorem [3]. We therefore assume that the Sylow 2-subgroups of $G$ are infinite. Let $P$ be a Sylow 2-subgroup and $K$ a procyclic normal subgroup of $P$. Choose an open normal subgroup $U$ of $G$ such that $U \cap P \leq K$. Then all Sylow 2-subgroups of $U$ are procyclic. Note that finite groups with cyclic Sylow 2-subgroups are soluble [4, Theorem 7.6.1] and therefore $U$ is prosoluble. The proof is complete. 
Recall that the Fitting height of a finite soluble group $G$ is the length $h(G)$ of a shortest series of normal subgroups all of whose quotients are nilpotent. By the Fitting height of a prosoluble group $G$ we mean the length $h(G)$ of a shortest series of normal subgroups all of whose quotients are pronilpotent. Note that in general a prosoluble group does not necessarily have such a series. The parameter $h(G)$ is finite if, and only if, $G$ is an inverse limit of finite soluble groups of bounded Fitting height. The next lemma follows from the corresponding result on finite soluble groups (see for example [2]).

LEMMA 2.8. Let $p$ be a prime, $h$ a positive integer, and $G$ a prosoluble group having a Hall $p^{\prime}$-subgroup $K$ such that $h(K)=h$. Then $h(G)$ is finite and bounded in terms of $h$ only.

\section{Auxiliary results}

In this section we develop the common part of the proofs of Theorem 1.1 and Theorem [1.2, We start though with two remarks underlining the difference between the theorems - the fact that abstract locally finite subgroups in a group satisfying the hypothesis of Theorem 1.1 are finite while those in Theorem 1.2 may be infinite.

REMARK 3.1. Because of Theorem 2.2 in a profinite group with virtually torsion-free centralizers all abstract locally finite subgroups are finite. Thus, if $G$ is as in Theorem 1.1 then the abstract locally finite subgroups of $G$ are finite.

REMARK 3.2. If $G$ is as in Theorem 1.2, that is, a profinite group in which the centralizer of every nontrivial element is virtually procyclic, then $G$ can have infinite locally finite subgroups. On the other hand, it is easy to see that for any prime $p$ the abstract locally finite p-subgroups of $G$ are finite.

The next two lemmas provide key technical tools for the proofs of Theorem 1.1 and Theorem 1.2 respectively. Though the statements of the lemmas are different, the proofs are almost identical so we give a common proof for both.

LEMmA 3.3. Let $G$ be as in Theorem 1.1, that is, a profinite group in which the centralizer of every nontrivial element is virtually torsionfree while the centralizer of every element of infinite order is virtually procyclic. Suppose that $G=H A$, where $A \cong \mathbb{Z}_{p}$ and $H$ a normal pro- $p^{\prime}$ subgroup. Then $G$ is virtually procyclic.

LEMMA 3.4. Let $G$ be as in Theorem 1.2, that is, a profinite group in which the centralizer of every nontrivial element is virtually procyclic. 
Suppose that $G=H A$, where $H$ is a normal pro-p subgroup and $A$ an infinite procyclic pro-p $p^{\prime}$ subgroup. Then $G$ is virtually procyclic.

Proof of Lemma 3.3 and Lemma 3.4. Let $A_{i}=A^{i !}$ denote the subgroup generated by the $i$ th powers of elements of $A$. Set $H_{i}=$ $C_{H}\left(A_{i}\right)$ for $i=1,2, \ldots$ Each subgroup $H_{i}$ is virtually procyclic. Lemma 2.6 shows that the second commutator subgroup $H_{i}^{\prime \prime}$ is finite. Therefore the abstract subgroup $H_{0}=\bigcup_{i} H_{i}^{\prime \prime}$ is locally finite. By virtue of Remark 3.1 and Remark 3.2, $H_{0}$ is finite. Choose an open normal subgroup $M_{1}$ of $G$ such that $M_{1} \cap H_{0}=1$. It is sufficient to show that $M_{1}$ is virtually procyclic. We can work with the group $M_{1} A$ in place of $G$ and without loss of generality assume that $H_{0}=1$, that is, all centralizers $H_{i}$ are metabelian.

Suppose $N$ is an open normal $A$-invariant subgroup of $H$. Since $H / N$ is finite, there is an index $i$ such that $A_{i}$ acts trivially on $H / N$. Lemma 2.3 (iii) shows that $H=N H_{i}$. Recall that $H_{i}$ is metabelian. Hence $H / N$ is metabelian for any open normal $A$-invariant subgroup $N$ of $H$. We conclude that $H$ is metabelian. Due to the hypothesis on centralizers in $G$, it follows that $H^{\prime}$ is an abelian virtually procyclic group. In the next paragraph we will deal with the case where $H^{\prime}$ is finite. For now assume that $H^{\prime}$ is infinite. Write $H^{\prime}=K \times T$ where $T$ is a characteristic finite subgroup and $K$ a torsion-free procyclic subgroup. Choose an open normal subgroup $M_{2}$ of $G$ such that $M_{2} \cap$ $T=1$. It is sufficient to show that $M_{2}$ is virtually procyclic. We can work with the group $M_{2} A$ in place of $G$ and without loss of generality assume that $T=1$, that is, $H^{\prime}$ is torsion-free procyclic. Let $L$ be a subgroup of $H^{\prime}$ isomorphic to $\mathbb{Z}_{q}$ for a prime $q$ (here $q \neq p$ in the case of Lemma 3.3 and $q=p$ in the case of Lemma 3.4). Since the group of automorphisms of $\mathbb{Z}_{q}$ does not contain infinite pro- $q^{\prime}$ subgroups, a subgroup of finite index $B \leq A$ centralizes $L$. It is sufficient to show that $H B$ is virtually procyclic and so without loss of generality we assume that $A$ centralizes $L$. Then of course $[H, A]$ centralizes $L$. Recall that $H=[H, A] C_{H}(A)$. Note that $C_{H}(A)$ is virtually procyclic and so the index of the centralizer of $L$ in $C_{H}(A)$ is finite. Combining this with the facts that $[H, A]$ centralizes $L$ and $H=[H, A] C_{H}(A)$, we deduce that the index $\left[G: C_{G}(L)\right]$ is finite. Since $C_{G}(L)$ is virtually procyclic, $G$ is virtually procyclic, too. Thus, in the case where $H^{\prime}$ is infinite the group $G$ is virtually procyclic.

We now deal with the case where $H^{\prime}$ is finite. Passing to an open normal subgroup we may assume that $H$ is abelian. If $H$ is finite, the lemmas hold. So we assume that $H$ is infinite. It follows that $H$ is 
virtually procyclic and we can write $H$ as a direct product of a torsionfree procyclic subgroup and a finite subgroup. Choose an open normal subgroup $M_{3}$ of $G$ such that $M_{3} \cap H$ is torsion-free procyclic. As above, work with the group $M_{3} A$ in place of $G$ and without loss of generality assume that $H$ is torsion-free procyclic. Again, we consider a pro- $q$ subgroup $L$ of $H$ and see that its centralizer $C_{G}(L)$ has finite index in $G$ and is virtually procyclic. The proof is complete.

We can now reduce both Theorem 1.1 and Theorem 1.2 to questions on prosoluble groups.

LEMma 3.5. Let $G$ be either as in Theorem 1.1 or as in Theorem 1.2. Then $G$ is virtually prosoluble.

Proof. Assume that $G$ is not virtually prosoluble. By virtue of the Feit-Thompson theorem, the Sylow 2-subgroups of $G$ are infinite. Moreover, by Lemma [2.5, for at least one odd prime $q \in \pi(G)$ the Sylow $q$-subgroups of $G$ are infinite.

Let $P$ be a Sylow 2-subgroup and $Q$ a Sylow $q$-subgroup of $G$. Since the subgroups $P$ and $Q$ are infinite, we can choose an infinite chain of open normal subgroups $G=N_{1}>N_{2}>\ldots$ such that $P \cap N_{i}>P \cap N_{i+1}$ and $Q \cap N_{i}>Q \cap N_{i+1}$ for each $i=1,2, \ldots$

Set $P_{i}=P \cap N_{i}$ and $K_{i}=N_{G}\left(P_{i}\right)$. The Frattini argument shows that $G=N_{i} K_{i}$ for each $i=1,2, \ldots$ Suppose first that the Sylow $q$-subgroups $Q_{i}$ of $K_{i}$ are torsion for each $i=1,2, \ldots$ Obviously, the subgroups $Q_{i}$ can be chosen in such a way that $Q_{1} \leq Q_{2} \leq \ldots$. Let $Q_{0}=\cup_{i} Q_{i}$ and $N=\cap_{i} N_{i}$. The choice of the chain $G=N_{1}>N_{2}>\ldots$ guarantees that $Q \cap N$ has infinite index in $Q$. Therefore $Q_{0}$ is an infinite (abstract) locally finite subgroup of $G$. In view of Remark 3.1 (or Remark 3.2) this is a contradiction. Hence, there is an idex $j$ such that $Q_{j}$ is not torsion. Choose an infinite procyclic subgroup $A \leq Q_{j}$. Since $A$ normalizes $P_{j}$, Lemma 3.3 shows that $P_{j}$ is virtually procyclic. Now Lemma 2.7 says that the subgroup $N_{j}$ is virtually prosoluble. Of course, this implies that also $G$ is virtually prosoluble. This completes the proof.

\section{Theorem 1.1}

In this section we prove Theorem 1.1. Thus, throughout this section $G$ is as in Theorem 1.1, that is, a profinite group in which the centralizer of every nontrivial element is virtually torsion-free while the centralizer of every element of infinite order is virtually procyclic. 
LEMma 4.1. Suppose that $G$ is prosoluble, written as a product $G=P H$, where $P$ is a pro-p subgroup and $H$ is a virtually procyclic pro-p $p^{\prime}$ subgroup. Then $G$ is either virtually pro-p or virtually procyclic.

Proof. First, we observe that $H$ is a Hall $p^{\prime}$-subgroup of $G$. By Lemma $2.8 G$ has a finite series

$$
1=G_{0} \leq G_{1} \leq G_{2} \leq \cdots \leq G_{s+1}=G
$$

of characteristic subgroups such that each quotient $G_{u+1} / G_{u}$ is either a pro- $p$ group or a pro- $p^{\prime}$ group. Note that $G_{s}=P_{0} H_{0}$, where $P_{0}=P \cap G_{s}$ and $H_{0}=H \cap G_{s}$ (see for example [9, Lemma 2.4]). Arguing by induction on the length of the above series we can assume that $G_{s}$ is either virtually pro- $p$ or virtually procyclic. If at least one of the groups $G_{s}, G / G_{s}$ is finite, the result is immediate so we assume that both $G_{s}$ and $G / G_{s}$ are infinite.

Suppose first that $G_{s}$ is virtually pro- $p$. Since the Hall $p^{\prime}$-subgroups of $G_{s}$ are finite, we can replace $G$ by its open subgroup whose intersection with $G_{s}$ is a pro- $p$ group and the intersection with $H$ is torsion-free procyclic. Therefore without loss of generality assume that $G_{s}$ is a pro$p$ group and $G / G_{s}$ is a torsion-free procyclic pro- $p^{\prime}$ group. Choose a nontrivial pro- $q$ subgroup $L$ in $G$, for $q \neq p$, and look at the product $G_{s} L$. Lemma 3.3 shows that $G_{s}$ is virtually procyclic. Let $K$ be an infinite characteristic procyclic subgroup of $G_{s}$. Since pro- $p^{\prime}$ groups of automorphisms of $\mathbb{Z}_{p}$ are finite, it follows that $C_{G}(K)$ has finite index in $G$ and is virtually procyclic, as required.

Now we need to deal with the case where $G_{s}$ is virtually procyclic. Suppose that $G / G_{s}$ is a pro- $p^{\prime}$ group. Then as above $G / G_{s}$ is virtually procyclic, too and $G=G_{s} H$. Replacing $H$ by a subgroup of finite index we can assume that $G / G_{s}$ is procyclic. Let again $K$ be a characteristic open procyclic subgroup of $G_{s}$. If $K$ is virtually pro- $p^{\prime}$, then the whole group $G$ is virtually pro- $p^{\prime}$ and so $G$ is virtually procyclic since $H$ is open in $G$. Otherwise, assume that $K$ contains an infinite pro- $p$ subgroup $K_{0}$. Since pro- $p^{\prime}$ groups of automorphisms of $\mathbb{Z}_{p}$ are finite, it follows that $C_{G}\left(K_{0}\right)$ has finite index in $G$. Taking into account that $C_{G}\left(K_{0}\right)$ is virtually procyclic, the result follows.

It remains to handle the situation where $G / G_{s}$ is a pro- $p$ group, in which case $G=G_{s} P$. Again we look at a characteristic open procyclic subgroup $K$ of $G_{s}$. If $K$ is virtually pro- $p$, then the whole group $G$ is virtually pro- $p$ and we have nothing to prove. Assume that $K$ is not virtually pro- $p$ and so $K$ contains an infinite pro- $q$ subgroup $K_{1}$ for some prime $q \neq p$. As before, we see that $C_{G}\left(K_{1}\right)$ has finite index in $G$. Taking into account that $C_{G}\left(K_{1}\right)$ is virtually procyclic, the result follows. 
LEMMA 4.2. If $G$ is pronilpotent, then $G$ is either pro-p for some prime $p$ or virtually torsion-free procyclic.

Proof. Suppose that $G$ is not pro- $p$. Observe that for any $p \in$ $\pi(G)$ the subgroup $O_{p^{\prime}}(G)$ is virtually torsion-free procyclic. Therefore $G$ is a direct product of two virtually torsion-free procyclic subgroups of coprime orders, whence the lemma follows.

Recall that a Sylow basis in a group $L$ is a family of pairwise permutable Sylow $p_{i}$-subgroups $P_{i}$ of $L$, exactly one for each prime. The basis normalizer of such Sylow basis in $L$ is the intersection of the normalizers of the $P_{i}$, that is, $\bigcap_{i} N_{L}\left(P_{i}\right)$. This subgroup is also known under the name of system normalizer. If $L$ is a profinite group and $T$ is a basis normalizer in $L$, then $T$ is pronilpotent and $L=\gamma_{\infty}(L) T$, where $\gamma_{\infty}(L)$ denotes the intersection of the terms of the lower central series of $L$. Furthermore, every prosoluble group $L$ possesses a Sylow basis and any two basis normalizers in $L$ are conjugate (see [11, Prop. 2.3.9] and [12, 9.2]).

We are now ready to complete the proof of Theorem 1.1 .

Proof of TheOrem 1.1. Recall that $G$ is a profinite group such that the centralizer of every nontrivial element is virtually torsion-free while the centralizer of every element of infinite order is virtually procyclic. We wish to prove that $G$ is either virtually pro- $p$ for some prime $p$ or virtually torsion-free procyclic. Because of Lemma 3.5 without loss of generality we can assume that $G$ is prosoluble. Choose a Sylow basis $P_{1}, P_{2}, \ldots$ in $G$. Set $G=K_{0}$ and $K_{i+1}=\gamma_{\infty}\left(K_{i}\right)$ for $i=0,1, \ldots$ Let $T_{i}$ denote the normalizer of the basis $P_{1} \cap K_{i}, P_{2} \cap K_{i}, \ldots$ in $K_{i}$. Thus, the subgroups $T_{i}$ are pronilpotent and $T_{i}$ normalizes $T_{j}$ whenever $i \leq j$. Combining Remark 3.1 with the property that $T_{i}$ normalizes $T_{j}$ whenever $i \leq j$ we derive that only finitely many of the subgroups $T_{i}$ are finite. Assume that the group $G$ is infinite and let $j$ be the minimal index such that $T_{j}$ is infinite. Since $T_{j}$ is infinite, Lemma 4.2 guarantees that we can pick an infinite procyclic pro- $p$ subgroup $A \leq T_{j}$. Recall that $T_{j}$ is a basis normalizer of $K_{j}$ and so $A$ normalizes a Sylow $q$-subgroup of $K_{j}$ for each $q \in \pi\left(T_{j}\right)$. Denote by $P$ a Sylow $p$-subgroup containing $A$ and by $H$ a Hall $p^{\prime}$-subgroup normalized by $A$ so that $G=P H$. In view of Lemma $3.3 H$ is virtually procyclic. The theorem now follows from Lemma 4.1. 


\section{Theorem 1.2}

In this section we will prove Theorem 1.2, Thus, throughout this section $G$ stands for a profinite group in which the centralizers of nontrivial elements are virtually procyclic.

LEMMA 5.1. If $G$ is prosoluble and $h(G)$ is finite, then $G$ is either virtually pro-p for some prime $p$ or virtually procyclic.

Proof. Use induction on $h=h(G)$. Suppose first that $h=1$, that is, $G$ is pronilpotent. Assume that $G$ is not pro- $p$. Observe that for any $p \in \pi(G)$ the subgroup $O_{p^{\prime}}(G)$ is virtually procyclic. Therefore $G$ is a direct product of two virtually procyclic subgroups of coprime orders, whence the lemma follows.

We now suppose that $h \geq 2$. Let $T$ be a basis normalizer in $G$ and $K=\gamma_{\infty}(G)$. Thus, $G=K T$. Since $T$ is pronilpotent and $h(K)=h-1$, by induction each of the subgroups $K$ and $T$ is either virtually pro- $p$ for some prime $p$ or virtually procyclic. We can choose an open normal subgroup $L$ in $G$ such that each of the subgroups subgroups $L_{1}=L \cap K$ and $L_{2}=L \cap T$ is either pro- $p$ for some prime $p$ or procyclic.

If both $L_{1}$ and $L_{2}$ are pro- $p$ for the same prime $p$, then $G$ is virtually pro- $p$ and we are done.

Suppose there are two different primes $p$ and $q$ such that $L_{1}$ is pro- $p$ while $L_{2}$ is pro- $q$. If $L_{2}$ has an infinite torsion-free procyclic subgroup, then by Lemma $3.4 L_{1}$ is procyclic. In that case $C_{G}\left(L_{1}\right)$ has finite index in $G$ (because pro- $p^{\prime}$ groups of automorphisms of a procyclic pro- $p$ group are finite). Since $C_{G}\left(L_{1}\right)$ is virtually procyclic, we are done. Otherwise, $L_{2}$ is torsion and so by Theorem 2.1 locally finite. In view of Remark $3.2 L_{2}$ is finite and hence $G$ is virtually pro- $p$.

Now assume that $L_{1}$ is pro- $p$ while $L_{2}$ is procyclic. Let $B$ be the maximal pro- $p^{\prime}$ subgroup of $L_{2}$. If $B$ is finite, then $G$ is virtually pro$p$. We therefore assume that $B$ is infinite. Lemma 3.4 tells us that $L_{1} B$ is virtually procyclic. In particular, $C_{B}\left(L_{1}\right)$ has finite index in $B$. Observe that $C_{B}\left(L_{1}\right)$ commutes with both $L_{1}$ and $L_{2}$. Therefore $L \leq C_{G}(B)$. Note that $C_{G}(B)$ is virtually procyclic, whence $G$ is virtually procyclic, as required.

Suppose $L_{1}$ is procyclic while $L_{2}$ is pro- $p$. If $L_{1}$ has a nontrivial pro- $q$ subgroup for a prime $q \neq p$, we argue as above and deduce that $G$ is virtually procyclic because pro- $q^{\prime}$ groups of automorphisms of a procyclic pro- $q$ group are finite. If $L_{1}$ is pro- $p$, the group $G$ is virtually pro- $p$.

It remains to consider the case where both $L_{1}$ and $L_{2}$ are procyclic while $L_{1}$ is not pro- $p$ (not even virtually pro- $p$ ). If $L_{1}$ has a finite 
subgroup $M$, we deduce that $G$ is virtually procyclic since $C_{G}(M)$ has finite index in $G$. Thus, assume that $L_{1}$ is torsion-free. Choose two subgroups $A_{1}, A_{2} \leq L_{1}$ where $A_{1} \cong \mathbb{Z}_{p}$ and $A_{2} \cong \mathbb{Z}_{q}$ for different primes $p$ and $q$. We can assume that both $C_{G}\left(A_{1}\right)$ and $C_{G}\left(A_{2}\right)$ have infinite index in $G$. Let $B_{1}$ and $B_{2}$ be the Sylow $p$ - and $q$-subgroups of $L_{2}$, respectively. It follows that both $B_{1}$ and $B_{2}$ are infinite (since $C_{G}\left(A_{1}\right)$ and $C_{G}\left(A_{2}\right)$ have infinite index). Moreover, $B_{1} \cap C_{G}\left(A_{1}\right)=1$ and $B_{2} \cap C_{G}\left(A_{2}\right)=1$. Furthermore, $C_{B_{2}}\left(A_{1}\right)$ is infinite. Thus, $C_{G}\left(A_{1}\right)$ contains the subgroup $A_{2} C_{B_{2}}\left(A_{1}\right)$, which is not virtually procyclic. This contradiction completes the proof.

We will require the celebrated theorem of Thompson [15 that says that if a finite soluble group $K$ admits a coprime automorphism $\phi$ of prime order, then $h(K)$ is bounded in terms of $h\left(C_{K}(\phi)\right)$ only (see [16] for a survey on results of similar nature).

If $K$ is a locally finite group, we write $h(K) \leq h$ to mean that $K$ has a normal series of length at most $h$ all of whose factors are locally nilpotent. It is well-known that if $K$ is a locally finite-soluble group, then $h(K) \leq h$ if and only if $h(U) \leq h$ for every finite subgroup $U \leq K$.

We will now complete the proof of Theorem 1.2 .

Proof of Theorem 1.2. Assume that $G$ is infinite. Further, taking into account Lemma 3.5 without loss of generality we assume that $G$ is prosoluble. In view of Lemma 5.1 it is sufficient to show that $h(G)$ is finite. Choose a Sylow basis $P_{1}, P_{2}, \ldots$ in $G$. Set $G=K_{0}$ and $K_{i+1}=\gamma_{\infty}\left(K_{i}\right)$ for $i=0,1, \ldots$ Let $T_{i}$ denote the normalizer of the basis $P_{1} \cap K_{i}, P_{2} \cap K_{i}, \ldots$ in $K_{i}$. Thus, the subgroups $T_{i}$ are pronilpotent and $T_{i}$ normalizes $T_{j}$ whenever $i \leq j$. Suppose that a Sylow $p$-subgroup of some $T_{j}$ is non-torsion. Then we can pick an infinite procyclic pro- $p$ subgroup $A \leq T_{j}$. Recall that $T_{j}$ is a basis normalizer of $K_{j}$ and so $A$ normalizes a Hall $p^{\prime}$-subgroup $H$ of $K_{j}$. In view of Lemma $3.4 H$ is virtually procyclic. Therefore Lemma 2.8 shows that $h(G)$ is finite, as required.

Hence, we only need to deal with the case where all Sylow subgroups of the $T_{i}$ are torsion. In particular, because of Theorem 2.1, the Sylow subgroups of the $T_{i}$ are locally finite. Remark 3.2 now implies that the Sylow subgroups of the $T_{i}$ are finite. Let $D_{i}$ denote the abstract subgroup generated by all Sylow subgroups of $T_{i}$. Observe that $D_{i}$ is locally finite and $D_{i}$ normalizes $D_{j}$ whenever $i \leq j$. Therefore the subgroup $D=\left\langle D_{i} ; i=1,2, \ldots\right\rangle$ is a locally finite-soluble group with finite Sylow subgroups. Choose an element $x \in D$ of prime order $p$. Since $D$ is residually finite and the Sylow $p$-subgroup of $D$ is finite, there is a normal $p^{\prime}$-subgroup $N$ of finite index in $D$. By hypotheses 
$C_{N}(x)$ has a locally cyclic subgroup of finite index. Thus, there is $h$ such that $C_{N}(x) \leq h$. The Thompson Theorem [15] now tells us that there is $h_{0}$ such that $h(N) \leq h_{0}$. Since $N$ has finite index in $D$, we conclude that there is $h_{1}$ such that $h(D) \leq h_{1}$. Obviously, this implies that $D_{h_{1}}=1$ and we immediately obtain that $T_{h_{1}}=1$. Therefore $h(G) \leq h_{1}$, as requred. The proof is complete.

We will now deduce Corollary 1.3. Thus, assume that $G$ is a profinite group in which the centralizer of every nontrivial element is virtually procyclic and suppose that $G$ is not a pro- $p$ group. We wish to show that $G$ has finite rank. Obviously, this is correct if $G$ is virtually procyclic so, by virtue of Theorem 1.2 , we may assume that $G$ is virtually pro- $p$ for some prime $p$. We also assume that $G$ is infinite. Let $P$ be the largest normal pro- $p$ subgroup in $G$ and $a \in G$ an element of prime order $q \neq p$. Such an element $a$ exists since $G$ is not a pro- $p$ group. We now require the following theorem, due to Khukhro [8].

THEOREM 5.2. Let $K$ be a finite nilpotent group with an automorphism $\alpha$ of prime order $q$ such that $C_{K}(\alpha)$ has rank $r$. Then $K$ contains a characteristic subgroup $N$ such that $N$ has q-bounded nilpotency class and $K / N$ has $(q, r)$-bounded rank.

Using the standard inverse limit argument we deduce that if $K$ is a pronilpotent group admitting a coprime automorphism $\alpha$ of prime order $q$ such that $C_{K}(\alpha)$ has rank $r$, then $K$ contains a characteristic subgroup $N$ such that $N$ is nilpotent (with $q$-bounded nilpotency class) and $K / N$ has $(q, r)$-bounded rank.

Coming back to our group $G$, observe that $a$ induces an automorphism of $P$ of order dividing $q$. Since $C_{P}(a)$ is virtually procyclic, it follows that $C_{P}(a)$ has finite rank and therefore $P$ has a characteristic subgroup $N$ such that $N$ is nilpotent and $P / N$ has $(q, r)$-bounded rank. Since $N$ is nilpotent, it is contained in the centralizer of any nontrivial element of its centre. The centralizer is virtually procyclic and so both $N$ and $P / N$ have finite rank. We conclude that $P$ (and therefore $G$ ) has finite rank, as required.

\section{References}

[1] J. Alonso, T. Brady, D. Cooper, V. Ferlini, M. Lustig, M. Mihalik, M. Shapiro, and H. Short, Notes on word hyperbolic groups. In: Short, H.B., ed. Group Theory from a Geometrical Viewpoint, Proc. ICTP Trieste, World Scientific Publishing Co., Inc., River Edge, NJ, pp. 3-63.

[2] C. Casolo, E. Jabara, P. Spiga, On the Fitting height of factorised soluble groups, J. Group theory, 17, 911-17924. 
[3] W. Feit and J. Thompson, Solvability of groups of odd order, Pacific J. Math., 13 (1963), 775-1029.

[4] D. Gorenstein, Finite groups, Chelsea Publishing Co., New York, 1980.

[5] Hall, P., Kulatilaka, C. R.: A property of locally finite groups. J. London Math. Soc. 39 (1964), 235-239.

[6] B. Hartley, Centralizers in locally finite groups, in: "Group Theory, Bressanone, 1986", in: Lecture Notes in Math., vol.1281, Springer, Berlin, 1987, pp. 36-1751.

[7] O.H. Kegel, B.F.A. Wehrfritz, Locally finite groups, North-Holland, Amsterdam, 1973.

[8] E. I. Khukhro, Groups with an automorphism of prime order that is almost regular in the sense of rank, J. Lond. Math. Soc. (2), 77 (2008), no. 1, 130-17148.

[9] E. I. Khukhro, P. Shumyatsky, On the length of finite factorized groups, Annali di Matematica, 194 (2015), 1775171780.

[10] I. Kapovich and D Wise, The equivalence of some residual properties of word-hyperbolic groups. J. Algebra 223 (2000)

[11] L. Ribes and P. Zalesskii, Profinite groups, Springer, Berlin, 2000.

[12] D. Robinson, A Course in the Theory of Groups, Springer, New York, 1996.

[13] P. Shumyatsky, P. Zalesskii, T. Zapata, Profinite groups in which centralizers are abelian, Israel J. Math., 230 (2019), 831-854.

[14] P. Shumyatsky, Profinite groups with pronilpotent centralizers, Israel J. Math., to appear.

[15] J. G. Thompson, Automorphisms of solvable groups, J. Algebra 1 (1964), 259-267.

[16] A. Turull, Character theory and length problems, in "Finite and locally finite groups (Istanbul, 1994)", 37717400, NATO Adv. Sci. Inst. Ser. C Math. Phys. Sci., 471, Kluwer Acad. Publ., Dordrecht, 1995.

[17] E. Zelmanov, On periodic compact groups, Israel J. Math., 77 (1992), 83-95.

Department of Mathematics, University of Brasilia, Brazil

E-mail address: pavel@unb.br, pz@mat.unb.br 